Pacific Journal of Mathematics

QUATERNION ORDERS ASSOCIATED WITH TERNARY 


\title{
QUATERNION ORDERS ASSOCIATED WITH TERNARY LATTICES
}

\author{
GORDON L. NIPP
}

It is advantageous for the study of the spinor genus of quaternion orders to realize each order as corresponding to a ternary lattice. In the two known correspondences, those of Eichler and Pall, the question of whether the mapping is onto or not is not considered. Peters has investigated the question for Eichler's correspondence, and his results show that it is not onto. Pall's correspondence, though onto, is only defined over the rational integers. In this article, a generalization to Dedekind domains of Pall's correspondence is defined. Those orders which are images of ternary lattices under the correspondence are completely determined, and the relationship of this mapping to Eichler's is examined.

1. Introduction. The terminology will be that of O'Meara [4]. Throughout we will be dealing with a regular ternary quadratic space $V$ over the quotient field $F$ (characteristic of $F$ not equal to 2) of a Dedekind domain $D$ and with the even Clifford algebra $C^{+}=C^{+}(V)$ of $V$. For convenience we will assume that $F$ is a global field.

The content of the individual sections is as follows:

In $\S 2$ we give some necessary preliminary results on the relationship between a regular ternary quadratic space, its even Clifford algebra, and their rotations.

We define a mapping $\vartheta$ from an integral ternary lattice $L$ on $V$ to an order $\vartheta_{L}$ on the quaternion algebra $C^{+}(V)$ in $\S 3 . \vartheta_{L}$ is the $D$-module in $C^{+}$generated over $D$ by 1 and all products $x y$ such that $x$ and $y$ are in $L$. We also determine in this section that the mapping is one-to-one and that two integral ternary lattices $L$ and $K$ are in the same class (spinor genus, genus) if and only if the orders $\vartheta_{L}$ and $\vartheta_{K}$ are in the same class (spinor genus, genus).

The problem of whether our mapping is onto or not is formulated in the following two contexts:

(i) If $\vartheta$ is an order on the even Clifford algebra of a fixed regular ternary quadratic space $V$, does there exist an integral lattice $L$ on $V$ such that $\vartheta_{L}=\vartheta$ ?

(ii) If $\vartheta$ is an order on a quaternion algebra, does there exist a regular ternary space $V$ and an integral lattice $L$ on $V$ such that $\vartheta_{L} \approx \vartheta ?$

In $\S 4$ we give a counterexample to answer the first question, but we do show that if an order $\vartheta_{L}$ is the image of a lattice $L$, then all orders in the genus of $\vartheta_{L}$ are images as well. 
In $\S 5$ we show that a necessary and sufficient condition that the second question be answered in the affirmative is that the square root of the volume of $\vartheta$ be a square in the ideal class group of $D$. This condition is satisfied in many important cases (e.g., it is always satisfied over the rational integers, or, more generally, when $\vartheta$ is free) and yields one of the principal advantages of our mapping over Eichler's. (It can be seen from [6], §4, that Eichler's correspondence is not onto in these cases.) Additionally, we investigate the relationship between those orders which are images under Eichler's correspondence and those which are images under our mapping.

Some of the material in this article is from a $\mathrm{Ph}$. D. dissertation written at the University of Southern California under Professor Dennis Estes. The author wishes to gratefully acknowledge Professor Estes' advice and encouragement.

2. Clifford algebras. Let $D$ be a Dedekind domain whose quotient field $F$ is a global field (char $F \neq 2$ ). Let $V$ be a regular ternary quadratic space over $F$ with associated quadratic form $\widetilde{N}$ and symmetric bilinear form $\widetilde{B}$, and let $C^{+}$be the even Clifford algebra of $V$. We assume that the vectors $x_{1}, x_{2}, x_{3}$ form a basis for $V$ with $\widetilde{B}\left(x_{i}, x_{j}\right)=a_{i j} \in F$. The relations

$$
x^{2}=\tilde{N}(x) \cdot 1, x y+y x=2 \widetilde{B}(x, y) \cdot 1 \quad \text { for all } x, y \in V
$$

imply that the products

$$
1, \alpha_{1}=x_{2} x_{3}, \alpha_{2}=x_{3} x_{1}, \alpha_{3}=x_{1} x_{2}
$$

form a basis for $C^{+}$over $F$, which we shall call the basis for $C^{+}$ corresponding to $x_{1}, x_{2}, x_{3}$. These basis elements are multiplied as follows:

$$
\begin{gathered}
\alpha_{i}^{2}=-a_{j j} a_{k k} \cdot 1+2 a_{j k} \alpha_{i} \\
\alpha_{i} \alpha_{j}=\left\{\begin{array}{l}
2 a_{i j} a_{k k} \cdot 1-a_{k k} \alpha_{k} ; \sigma \text { even } \\
a_{k k} \alpha_{k}+2 a_{i k} \alpha_{i}+2 a_{j k} \alpha_{j}-4 a_{i k} \alpha_{j k} \cdot 1 ; \sigma \text { odd }
\end{array}\right.
\end{gathered}
$$

for each permutation

$$
\sigma=\left(\begin{array}{lll}
1 & 2 & 3 \\
i & j & k
\end{array}\right)
$$

It is well-known that $C^{+}$is a quaternion algebra. Conjugates of the basis elements take the form

$$
\bar{\alpha}_{i}=-\alpha_{i}+2 a_{j k} \cdot 1 \text {. }
$$

Furthermore, if $N$ and $B$ are the quadratic and symmetric bilinear 
forms on $C^{+}$,

$$
\begin{aligned}
N\left(\alpha_{i}\right) & =\alpha_{i} \bar{\alpha}_{i}=a_{j j} a_{k k} \cdot 1 \\
B\left(\alpha_{i}, 1\right) & =\frac{1}{2}\left(\alpha_{i}+\bar{\alpha}_{i}\right)=a_{j k} \cdot 1 \\
B\left(\alpha_{\imath}, \alpha_{j}\right) & =\frac{1}{2}\left(\alpha_{i} \bar{\alpha}_{j}+\alpha_{j} \bar{\alpha}_{i}\right)=\left(2 a_{i k} a_{j k}-a_{k k} a_{i j}\right) \cdot 1 .
\end{aligned}
$$

It is natural to ask about the relationship between bases for $C^{+}$ corresponding to different bases of $V$. Let $1, \alpha_{1}, \alpha_{2}, \alpha_{3}$ and $1, \beta_{1}, \beta_{2}$, $\beta_{3}$ be the bases for $C^{+}$corresponding to the bases $x_{1}, x_{2}, x_{3}$ and $y_{1}, y_{2}$, $y_{3}$ for $V$ respectively. Suppose $\widetilde{B}\left(x_{i}, x_{j}\right)=a_{i j}$ and $\widetilde{B}\left(y_{i}, y_{j}\right)=b_{i j}$, and let $T$ be the change of basis matrix satisfying

$$
\left(y_{1}, y_{2}, y_{3}\right)^{\prime}=T\left(x_{1}, x_{2}, x_{3}\right)^{\prime} \text {. }
$$

(The prime denotes transpose.) It is most useful to state our result in terms of the bases for $C^{+}$

$$
\delta=\left(1, \delta_{1}, \delta_{2}, \delta_{3}\right), \rho=\left(1, \rho_{1}, \rho_{2}, \rho_{3}\right)
$$

where for $i \neq j, j \neq k, k \neq i$

$$
\delta_{i}=\alpha_{i}-\alpha_{j k} \cdot 1 \text { and } \rho_{i}=\beta_{i}-b_{j k} \cdot 1 .
$$

The proof of the following proposition is computational and has been carried through over the rational integers by Pall in [5], Theorem 1.

Proposition 2.1. The bases $\delta$ and $\rho$ of $C^{+}$are related by

$$
\rho=\delta \cdot\left[\begin{array}{cccc}
1 & 0 & 0 & 0 \\
0 & & & \\
0 & \operatorname{adj} & T & \\
0 & & &
\end{array}\right]
$$

where adj $T$ is the adjoint of $T$.

We note that it is a consequence of Proposition 2.1 that the discriminants of $V$ and $C^{+}(V)$ in corresponding bases satisfy

$$
d\left(1, \alpha_{1}, \alpha_{2}, \alpha_{3}\right)=d^{2}\left(x_{1}, x_{2}, x_{3}\right) .
$$

Much is known about the relationship between the rotations of $V$ and of $C^{+}(V)$ (see [2], §4.2). Some of the development will be sketched here for later reference. Let $\sigma$ be a member of the group of rotations $O^{+}(V)$ of the regular ternary space $V$. It is well-known that $\sigma$ can be expressed as the product of two symmetries $\sigma=\tau_{u} \tau_{v}$ where $u, v \in V$. In the Clifford algebra of $V$, 


$$
\sigma(x)=\tau_{u} \tau_{v}(x)=(u v) x(u v)^{-1}
$$

for every $x \in V$. We shall define a mapping $\dot{\phi}: C^{+} \rightarrow C^{+}$which is induced in a natural way from $V$ on $C^{+}$by $\sigma$. If the vectors $x_{1}, x_{2}$, $x_{3}$ form a basis for $V, \phi$ is the linear mapping defined on a basis for $C^{+}$by

$$
\dot{\phi}\left(x_{i} x_{j}\right)=\sigma\left(x_{i}\right) \sigma\left(x_{j}\right), \dot{\phi}(1)=1 .
$$

So for each $\eta \in C^{+}$,

$$
\phi(\eta)=(u v) \eta(u v)^{-1} \text {. }
$$

$\phi$ is clearly an isometry, and it follows from Proposition 2.1 that $\phi$ is a rotation. So $\phi \in O^{+}\left(C^{+}\right)$.

Conversely, if $\phi \in O^{+}\left(C^{+}\right)$with $\phi(1)=1$, then, since $C^{+}$is a quaternion algebra, there exists $\xi \in C^{+}$with $N(\xi) \neq 0$ such that $\phi(\eta)=$ $\xi \eta \xi^{-1}$ for every $\eta \in C^{+}$. We infer from [2], $\S 5.2$, that the mapping $\sigma: V \rightarrow V$ defined by $\sigma(x)=\xi x \xi^{-1}$ satisfies the conditions of the following proposition:

Proposition 2.2. Let $\phi \in O^{+}\left(C^{+}\right)$with $\phi(1)=1$. Then there exists a mapping $\sigma \in O^{+}(V)$ such that $\phi$ is the mapping induced on $C^{+}$by $\sigma$.

It follows from Proposition 2.2 that every element $\xi$ of $C^{+}$can be expressed as $\xi=u_{1} u_{2}$ where $u_{1}$ and $u_{2}$ are in $V$.

3. The correspondence. If $L$ is a lattice on $V$, it is well-known that there exists a basis $x_{1}, x_{2}, x_{3}$ for $V$ and fractional ideals $A_{1}, A_{2}$, $A_{3}$ of $D$ in $F$ such that

$$
L=A_{1} x_{1}+A_{2} x_{2}+A_{3} x_{3} .
$$

We assume that $L$ is an integral lattice on $V(N(L) \subset D)$, and with $L$ we associate the $D$-module $\vartheta_{L}$ in $C^{+}$generated over $D$ by 1 and all products $x y$ such that $x$ and $y$ are in $L$. Then

$$
\begin{aligned}
\vartheta_{L} & =D \cdot 1+\sum_{i, j=1}^{3} A_{i} A_{j} x_{i} x_{j} \\
& =D \cdot 1+\left[\sum_{i=1}^{3} A_{i}^{2} x_{i}^{2}+A_{3} A_{2}\left(-x_{2} x_{3}+2 \widetilde{B}\left(x_{3}, x_{2}\right) \cdot 1\right)\right. \\
& \left.+A_{1} A_{3}\left(-x_{3} x_{1}+2 \widetilde{B}\left(x_{1}, x_{3}\right) \cdot 1\right)+A_{2} A_{1}\left(-x_{1} x_{2}+2 \widetilde{B}\left(x_{2}, x_{1}\right) \cdot 1\right)\right] \\
& +A_{2} A_{3} \alpha_{1}+A_{3} A_{1} \alpha_{2}+A_{1} A_{2} \alpha_{3} \\
& =D \cdot 1+\left[\sum_{i=1}^{3} A_{i}^{2} \widetilde{N}\left(x_{i}\right)+2 \sum_{i<j} A_{i} A_{j} \widetilde{B}\left(x_{i}, x_{j}\right)\right] \cdot 1 \\
& +A_{2} A_{3} \alpha_{1}+A_{3} A_{1} \alpha_{2}+A_{1} A_{2} \alpha_{3}
\end{aligned}
$$




$$
\begin{aligned}
& =D \cdot 1+\tilde{N}(L) \cdot 1+A_{2} A_{3} \alpha_{1}+A_{3} A_{1} \alpha_{2}+A_{1} A_{2} \alpha_{3} \\
& =D \cdot 1+A_{2} A_{3} \alpha_{1}+A_{3} A_{1} \alpha_{2}+A_{1} A_{2} \alpha_{3}
\end{aligned}
$$

where $1, \alpha_{1}, \alpha_{2}, \alpha_{3}$ is the basis for $C^{+}$corresponding to $x_{1}, x_{2}, x_{3}$. An order on $C^{+}$is a lattice on $C^{+}$which is closed under multiplication and which contains 1. (The class, genus, and spinor genus of an order are defined as for any other lattice.) To show that $\vartheta_{L}$ is an order, we need only show multiplicative closure, and to this end we will perform the computations for one case.

$$
\begin{aligned}
\left(A_{2} A_{3} \alpha_{1}\right)\left(A_{3} A_{1} \alpha_{2}\right)= & A_{3}^{2} A_{1} A_{2} \alpha_{1} \alpha_{2} \\
= & A_{3}^{2} A_{1} A_{2}\left[2 \widetilde{B}\left(x_{1}, x_{2}\right) \widetilde{N}\left(x_{3}\right) \cdot 1-\tilde{N}\left(x_{3}\right) \alpha_{3}\right] . \\
\subset & {\left[A_{3}^{2} \widetilde{N}\left(x_{3}\right)\right]\left[A_{1} A_{2}\left(2 \widetilde{B}\left(x_{1}, x_{2}\right)\right)\right] \cdot 1 } \\
& +\left[A_{3}^{2} \tilde{N}\left(x_{3}\right)\right] A_{1} A_{2} \alpha_{3}
\end{aligned}
$$

and since

$$
A_{3}^{2} \tilde{N}\left(x_{3}\right), A_{1} A_{2}\left(2 \widetilde{B}\left(x_{1}, x_{2}\right)\right) \subset \tilde{N}(L) \subset D,
$$

we have

$$
\left(A_{2} A_{3} \alpha_{1}\right)\left(A_{3} A_{1} \alpha_{2}\right) \subset D \cdot 1+A_{1} A_{2} \alpha_{3} \subset \vartheta_{L} .
$$

So $\vartheta_{L}$ is an order on $C^{+}$.

We next show that the mapping $\vartheta: L \rightarrow \vartheta_{L}$ from integral ternary lattices on $V$ to orders in the even Clifford algebra $C^{+}$of $V$ is oneto-one.

THeOREM 3.1. Let $L$ and $K$ be integral lattices on $V$. If $\vartheta_{L}=$ $\vartheta_{K}$, then $L=K$.

Proof. The theorem localizes. So we assume $D$ to be a principal ideal domain and lattices to be free. Let

$$
L=D x_{1}+D x_{2}+D x_{3} \text { and } K=D y_{1}+D y_{2}+D y_{3},
$$

and suppose $\left(y_{1}, y_{2}, y_{3}\right)^{\prime}=T\left(x_{1}, x_{2}, x_{3}\right)^{\prime}$ where $T$ is the nonsingular $3 \times$ 3 change of basis matrix. Let

$$
S_{L}=\left\{\omega \in \vartheta_{L}: B(\omega, 1)=0\right\},
$$

and define $\delta$ and $\rho$ as in $\S 2$, (1). Clearly, $\delta_{i} \in S_{L}$ and $\rho_{i} \in S_{K}$ for $i=$ $1,2,3$, and $S_{L}=S_{K}$. Hence

$$
S_{L}=D \delta_{1}+D \delta_{2}+D \delta_{3}=D \rho_{1}+D \rho_{2},+D \rho_{3} .
$$

From Proposition 2.1, $\left(\rho_{1}, \rho_{2}, \rho_{3}\right)=\left(\delta_{1}, \delta_{2}, \delta_{3}\right) \cdot \operatorname{adj} T$. Thus, adj $T$ is unimodular, $T$ is unimodular, and $L=K$. 
In the sections that follow, we shall compare our mapping to Eichler's correspondence. The quadratic forms on the even Clifford algebras of a ternary space $V$ as defined by O'Meara and Eichler differ by a factor of two. Using O'Meara's development, it is consistent to treat the order

$$
\tilde{N}(L)^{-1}\left[(\widetilde{N})(L)+A_{2} A_{3} x_{2} x_{3}+A_{3} A_{1} x_{3} x_{1}+A_{1} A_{2} x_{1} x_{2}\right]
$$

as the image of the lattice

$$
L=A_{1} x_{1}+A_{2} x_{2}+A_{3} x_{3}
$$

under Eichler's correspondence (see [2], p. 96).

Clearly, if $A \neq(0)$ is a fractional ideal of $D$ in $F$, then the images of the ternary lattices $L$ and $A L$ under Eichler's mapping coincide. Thus an order can be the image of lattices in different genera. The next results show that this does not occur with our mapping.

THEOREM 3.2. Two integral ternary lattices are in the same class if and only if the quaternion orders $\vartheta_{L}$ and $\vartheta_{K}$ are in the same class.

Proof. Let $\phi \in O^{+}\left(C^{+}\right)$. We may assume $\phi(1)=1$, since if $\phi(1)=$ $\zeta \neq 1, \zeta$ is a unit, and we consider $\zeta^{-1} \phi$. As in $\S 2$, let $\sigma \in O^{+}(V)$ induce $\phi$ on $C^{-}$. Then

$$
L=\sigma(K)
$$

if and only if

$$
\vartheta_{L}=\vartheta_{\sigma(K)}=\phi\left(\vartheta_{K}\right) .
$$

So $L \in \operatorname{cls} K$ if and only if $\vartheta_{L} \in \operatorname{cls} \vartheta_{K}$.

Theorem 3.2 holds in the local case. Hence we can record the following corollary:

COROLlary 3.3. Two integral ternary lattices $L$ and $K$ are in the same genus if and only if the orders $\vartheta_{L}$ and $\vartheta_{K}$ are in the same genus.

The next lemma is needed in order to prove the analogous theorem for spinor genera.

Lemma 3.4. If $\Sigma \in O^{\prime}(V)$, then the mapping $\Phi$ induced by $\Sigma$ on $C^{+}$is in $O^{\prime}\left(C^{+}\right)$. If $\Phi \in O^{\prime}\left(C^{+}\right), \Phi(1)=1$, then there exists $\Sigma \in O^{\prime}(V)$ such that $\Phi$ is the mapping induced on $C^{+}$by $\Sigma$. 
Proof. Let $\Sigma \in O^{\prime}(V)$. If $x \in V$, then in the Clifford algebra for $V$

$$
\Sigma(x)=\tau_{u} \cdot \tau_{v}(x)=\xi^{-1} x \xi
$$

where $\tau_{u}$ and $\tau_{v}$ are symmetries on $V, \xi^{-1}=u v \in C^{+}$, and $N(\xi) \neq 0$. If $\Phi$ is the automorphism induced on $C^{+}$by $\sigma$, then $\Phi(\beta)=\xi^{-1} \beta \xi$ for every $\beta \in C^{+}$. By hypothesis, $N(\xi)$ is a square in $F$. Hence the spinor norm of $\Phi$ is 1 ; i.e., $\Phi \in O^{\prime}\left(C^{+}\right)$.

Conversely, let $\Phi \in O^{\prime}\left(C^{+}\right)$with $\Phi(1)=1$. Then, for some $\xi \in C^{+}$ and for every $\beta \in C^{+}, \Phi(\beta)=\xi^{-1} \beta \xi$ where $N(\xi)$ is a nonzero square in $F$. The mapping defined by $\Sigma(x)=\xi^{-1} x \xi$ for $x \in V$ is in $O^{+}(V)$ and induces $\Phi$ on $C^{+}$. There exist $u_{1}$ and $u_{2}$ in $V$ such that $\xi=u_{1} u_{2}$, $\Sigma=\tau_{u_{1}} \tau_{u_{2}}$, and since $N(\xi)$ is a square, the spinor norm of $\Sigma$ is 1 .

THeORem 3.5. Two integral ternary lattices $L$ and $K$ are in the same spinor genus if and only if $\vartheta_{L}$ and $\vartheta_{K}$ are in the same spinor genus.

Proof. If $\sigma \in O^{+}(V)$ induces $\phi \in O^{+}\left(C^{+}\right)$, and if the localization $\sigma_{p} \in O^{+}\left(V_{p}\right)$ of $\sigma$ at $p$ induces $\phi_{p} \in O^{+}\left(C_{p}^{+}\right)$, then clearly $\dot{\phi}_{p}$ is the localization at $p$ of $\phi$. Additionally, if $\sigma_{p} \in O^{+}\left(V_{p}\right)$ induces the localization $\phi_{p} \in O^{+}\left(C_{p}^{+}\right)$of $\phi$ at $p$, then $\sigma_{p}$ is the localization of $\sigma$ at $p$. If $\Sigma_{p} \in$ $O^{\prime}\left(V_{p}\right)$ induces $\Phi_{p} \in O^{\prime}\left(C_{p}^{+}\right)$and if $L$ and $K$ are integral lattices on $V$, then

$$
L_{p}=\sigma_{p} \Sigma_{p} K_{p}
$$

at every $p$ if and only if

$$
\vartheta_{L_{p}}=\vartheta_{\sigma_{p} \tau_{p}} K_{p}=\phi_{p} \Phi_{p} \vartheta_{K_{p}}
$$

at every $p$. Hence $L \in \operatorname{spn} K$ if and only if $\vartheta_{L} \in \operatorname{spn} \vartheta_{K}$.

4. Ternary lattices. We next consider the question of whether our mapping is onto or not. That it is not when the question is posed in the context of a fixed space $V$ and its even Clifford algebra $C^{+}$can be seen from the following counterexample. Let $V$ be a three dimensional vector space over the field of rational numbers with basis $X=\left(x_{1}, x_{2}, x_{3}\right)$. Define a symmetric bilinear form on $V$ by $B\left(x_{i}, x_{j}\right)=$ 0 if $i \neq j$ and $B\left(x_{i}, x_{i}\right)=2$ for $i=1,2,3$. Let $\vartheta$ be the $Z$-module generated in $C^{+}$by the set

$$
\beta=\left(1, \frac{x_{2} x_{3}}{2}, x_{3} x_{1}, x_{1} x_{2}\right) .
$$

$\vartheta$ is an order, as may be easily seen by multiplying the basis elements together. 
The discriminant of $V$ in the basis $X, d_{V}(X)$, is 8 , and the discriminant of $\vartheta$ in the basis $\beta, d_{\vartheta}(\beta)$, is 16. Assuming that $\vartheta=\vartheta_{L}$, the image of a lattice $L$ on $V$, we let $Y$ be a basis for $L$ over the integers and $\gamma$ the corresponding basis for $\vartheta=\vartheta_{L}$. We note that

$$
d_{\vartheta}(\beta)=d_{\vartheta}(\gamma)=d_{V}(Y)^{2}
$$

and that

$$
d_{V}(Y)=a^{2} d_{V}(X)
$$

where $a$ is the determinant of the (rational) change of basis matrix from $X$ to $Y$. Thus

$$
16=d_{V}(Y)^{2}=a^{4} d_{V}(X)^{2}=64 a^{4} .
$$

So $a^{4}=1 / 4$. This is impossible over the rationals; hence $\vartheta$ is not the image of a lattice on $V$.

However, a useful theorem does hold in this context.

THEOREM 4.1. Let $\vartheta$ be an order in the genus of $\vartheta_{L}$, where $L$ is an integral lattice on $V$. Then there exists a lattice $K$ in the genus of $L$ such that $\vartheta=\vartheta_{K}$.

Proof. We are assuming that at every $p$ there exists $\phi_{p} \in O^{+}\left(C_{p}^{+}\right)$ such that $\vartheta_{p}=\dot{\phi}_{p}\left(\vartheta_{L_{p}}\right)$. Without loss of generality, we may assume that $\dot{\rho}_{p}(1)=1$. By Proposition 2.2, there exists $\sigma_{p} \in O^{+}\left(V_{p}\right)$ such that $\phi_{p}$ is the mapping induced on $C_{p}^{+}$by $\sigma_{p}$. Thus,

$$
\vartheta_{p}=\phi_{p}\left(\vartheta_{L_{p}}\right)=\vartheta_{\sigma_{p}\left(L_{p}\right)}=\vartheta_{K_{(p)}}
$$

where $K_{(p)}=\sigma_{p}\left(L_{p}\right)$. Since there exists a basis $\alpha_{1}, \alpha_{2}, \alpha_{3}, \alpha_{4}$ for $C^{+}$ such that

$$
\vartheta=A_{1} \alpha_{1}+A_{2} \alpha_{2}+A_{3} \alpha_{3}+A_{4} \alpha_{4}
$$

and

$$
\vartheta_{L}=B_{1} \alpha_{1}+B_{2} \alpha_{2}+B_{3} \alpha_{3}+B_{4} \alpha_{4},
$$

it follows that $\vartheta_{p}=\vartheta_{L_{p}}$ at all but possibly the finite number of spots which divide any of the fractional ideals $A_{i}$ or $B_{i}$ for $i=1,2,3,4$. So by Theorem 3.1, $K_{(p)}=L_{p}$ for all but a finite number of spots $p$. Therefore, there exists a lattice $K^{\prime}$ on $V$ such that $K_{p}^{\prime}=K_{(p)}$ for all $p$ (see [4], 81: 14). Then

$$
\vartheta_{p}=\vartheta_{K_{(p)}}=\left(\vartheta_{K^{\prime}}\right)_{p}
$$

for every $p$, and $\vartheta=\vartheta_{K^{\prime}}$. Clearly $K^{\prime}$ is in the genus of $L$. 
5. Quaternion orders. The problem of the preceding section may be reformulated in the following way. For a fixed order $\vartheta$ over $D$ on a quaternion algebra $\mathfrak{Q}$ over $F$, does there exist a regular ternary quadratic space $V$ over $F$ and an integral lattice $L$ on $V$ such that $\vartheta_{L} \approx \vartheta$ ?

Let us choose an orthogonal basis

$$
\gamma=\left(1, \gamma_{1}, \gamma_{2}, \gamma_{3}\right)
$$

for $\mathfrak{A}$ with $\gamma_{1}^{2}=d_{1} \cdot 1, \gamma_{2}^{2}=d_{2} \cdot 1\left(d_{1}, d_{2} \in F\right), \gamma_{1} \gamma_{2}=\gamma_{3}$, and $\gamma_{1} \gamma_{2}=-\gamma_{2} \gamma_{1}$. Suppose, for a fixed $p, \alpha=\left(1, \alpha_{1}, \alpha_{2}, \alpha_{3}\right)$ is a basis for $\vartheta_{p}$ over $D_{p}$ and $\tilde{\alpha}=\left(\widetilde{\alpha}_{0}, \widetilde{\alpha}_{1}, \widetilde{\alpha}_{2}, \widetilde{\alpha}_{3}\right)$ is the corresponding dual basis for $\vartheta_{p}^{\#}$, the dual of $\vartheta_{p}$. Let $\left(t_{i j}\right), i, j=0,1,2,3$, be the change of basis matrix from $\gamma$ to $\tilde{\alpha}$. Then if $B$ is the natural bilinear form on $\mathfrak{A}, B\left(\tilde{\alpha}_{0}, 1\right)=1$ and $B\left(\tilde{\alpha}_{i}, 1\right)=B\left(\gamma_{i}, 1\right)=0$ for $i=1,2,3$, and

$$
\widetilde{\alpha}=\gamma\left(t_{i j}\right)=\gamma\left[\begin{array}{cccc}
B\left(\widetilde{\alpha}_{0}, 1\right) & 0 & 0 & 0 \\
t_{10} & t_{11} & t_{12} & t_{13} \\
t_{20} & t_{21} & t_{22} & t_{23} \\
t_{30} & t_{31} & t_{32} & t_{33}
\end{array}\right]=\gamma\left[\begin{array}{cccc}
1 & 0 & 0 & 0 \\
t_{10} & & & \\
t_{20} & & T & \\
t_{30} & & &
\end{array}\right]
$$

where $T=\left(t_{i j}\right)$ for $i, j=1,2,3$.

Define

$$
\begin{aligned}
& \delta\left(1, \gamma_{1}, \gamma_{2}, \gamma_{3}\right)=N\left(\gamma_{1}\right) N\left(\gamma_{2}\right) \quad \text { and } \\
& \delta=\delta\left(\widetilde{\alpha}_{0}, \widetilde{\alpha}_{1}, \widetilde{\alpha}_{2}, \widetilde{\alpha}_{3}\right)=\operatorname{det} T \cdot \delta\left(1, \gamma_{1}, \gamma_{2}, \gamma_{3}\right) .
\end{aligned}
$$

$\delta\left(1, \gamma_{1}, \gamma_{2}, \gamma_{3}\right)^{2}$ is the discriminant of $\mathfrak{R}$ in the basis $\gamma$, and the volume of $\vartheta_{p}^{\sharp}, v\left(\vartheta_{p}^{\sharp}\right)$, is $\delta^{2} D_{p}$.

THeOREm 5.1. Let $\vartheta$ be an order on a quaternion algebra $\mathfrak{A}$. $A$ necessary and sufficient condition that there exists an integral ternary lattice $L$ with $\vartheta_{L} \approx \vartheta$ is that $\sqrt{\overline{v(\vartheta)}}$ be a square in the ideal class group of $D$.

Proof. Write $L=A_{1} x_{1}+A_{2} x_{2}+A_{3} x_{3}$ where $A_{1}, A_{2}, A_{3}$ are fractional ideals and $x_{1}, x_{2}, x_{3}$ form a basis for the space $V$ spanned by L. From $\S 3$, (1),

$$
\vartheta_{L}=D \cdot 1+A_{2} A_{3} x_{2} x_{3}+A_{3} A_{1} x_{3} x_{1}+A_{1} A_{2} x_{1} x_{2} .
$$

Then

$$
v\left(\vartheta_{L}\right)=A_{1}^{4} A_{2}^{4} A_{3}^{4} \cdot \operatorname{det}\left(\widetilde{B}\left(x_{i}, x_{j}\right)\right)^{2}=d_{0}^{2} A^{4}
$$

where $d_{0}=\operatorname{det}\left(\widetilde{B}\left(x_{i}, x_{j}\right)\right)$ and $A=A_{1} A_{2} A_{3}$. Thus $\sqrt{v\left(\vartheta_{L}\right)}=d_{0} A^{2}$ is a square in the ideal class group of $D$. 
To prove sufficiency, suppose $v(\vartheta)=d_{0}^{2} A^{4}$ where $d_{0} \in F$ and $A$ is a fractional ideal, and let $1, \gamma_{1}, \gamma_{2}, \gamma_{3}$ be an orthogonal basis for $\mathfrak{i}$ as in (1) above. Let

$$
K=\left\{\omega \in \vartheta^{\sharp}: B(\omega, 1)=0\right\}, L=(A \cdot K)^{d_{0}}
$$

where the superscript denotes scaling. $L$ is a ternary lattice on $F L=V^{d_{0}}$ where $V=F \gamma_{1}+F \gamma_{2}+F \gamma_{3}$. It will be shown that the order $\vartheta_{L}$ in $C^{+}\left(V^{d_{0}}\right)$ corresponding to $L$ is isomorphic to $\vartheta$. This assertion will follow if we prove that (i) $L$ is integral, (ii) $C^{+}\left(V^{d_{0}}\right)$ is isomorphic to $\mathfrak{A}$, and (iii) $\vartheta_{L}$ is locally isomorphic to $\vartheta$ at every $p$ under the restriction to $\vartheta_{L}$ of the localization of the isomorphism in (ii).

To prove (i), we observe that

$$
\tilde{N}(L)=d_{0} A^{2} N(K)=\sqrt{v(\vartheta)} \cdot N(K)=\sqrt{v(\vartheta)} \cdot N\left(\vartheta^{\sharp}\right),
$$

the last step following from [6], §3. By [3], Theorem 9,

$$
\sqrt{v\left(\vartheta_{p}\right)} \cdot N\left(\vartheta_{p}^{\sharp}\right) \subset N\left(\vartheta_{p}\right)=D_{p} \text {. }
$$

Hence $\tilde{N}\left(L_{p}\right) \subset D_{p}$ for each $p$, and $\tilde{N}(L) \subset D$.

For (ii), define $\psi: C^{+}\left(V^{d_{0}}\right) \rightarrow \mathfrak{A}$ to be the linear map with $\psi(1)=1$, $\psi(\alpha \beta)=d_{0} \alpha \bar{\beta}$ for $\alpha, \beta \in V^{d_{0}}$. $\psi$ is clearly one-to-one. To see that it is an algebra isomorphism, it will suffice to verify that it preserves multiplication on basis elements of $C^{+}\left(V^{d_{0}}\right)$. We will carry through the computations for one case. A basis for $C^{+}\left(V^{d_{0}}\right)$ is $\left(1, \beta_{1}, \beta_{2}, \beta_{3}\right)$ where $\beta_{1}=\gamma_{2} \gamma_{3}, \beta_{2}=\gamma_{3} \gamma_{1}$, and $\beta_{3}=\gamma_{1} \gamma_{2}$. Noting that, in $C^{+}\left(V^{d_{0}}\right)$, $\gamma_{2}^{2}=-d_{0} d_{2} \cdot 1$, then

$$
\begin{aligned}
\psi\left(\beta_{3} \beta_{1}\right) & =-d_{0} d_{2} \psi\left(\gamma_{1} \gamma_{3}\right)=-d_{0}^{2} d_{2} \gamma_{1} \bar{\gamma}_{3}=d_{0}^{2} d_{2} \gamma_{1} \gamma_{3} \\
& =d_{0}^{2} \gamma_{1} \cdot d_{2} \gamma_{3}=d_{0}^{2} \gamma_{1} \gamma_{2}^{2} \gamma_{3}=d_{0} \gamma_{1} \bar{\gamma}_{2} \cdot d_{0} \gamma_{2} \bar{\gamma}_{3} \\
& =\psi\left(\beta_{3}\right) \cdot \psi\left(\beta_{1}\right) .
\end{aligned}
$$

So $C^{+}\left(V^{d_{0}}\right) \approx \mathfrak{A}$.

(iii) We will show that $\psi_{p}\left(\vartheta_{L_{p}}\right)=\vartheta_{p}$ at each $p$. Using the notation of the beginning of this section, we write

$$
K_{p}=D_{p} \widetilde{\alpha}_{1}+D_{p} \widetilde{\alpha}_{2}+D_{p} \widetilde{\alpha}_{3}
$$

as a lattice on $V_{p}$, and if $A_{p}=a_{p} D_{p}$,

$$
L_{p}=D_{p} a_{p} \widetilde{\alpha}_{1}+D_{p} a_{p} \widetilde{\alpha}_{2}+D_{p} a_{p} \widetilde{\alpha}_{3}
$$

as a lattice on $V_{p}^{d_{0}}$. The order in $C^{+}\left(V_{p}^{d_{0}}\right)$ corresponding to $L_{p}$ is

$$
\vartheta_{L_{p}}=D_{p} \cdot 1+D_{p} a_{p}^{2} \widetilde{\alpha}_{2} \tilde{\alpha}_{3}+D_{p} a_{p}^{2} \widetilde{\alpha}_{3} \widetilde{\alpha}_{1}+D_{p} a_{p}^{2} \widetilde{\alpha}_{1} \tilde{\alpha}_{2},
$$

and its image $\psi_{p}\left(\vartheta_{L_{p}}\right)$ in $\mathfrak{U}_{p}$ is an order with basis 


$$
\left(1, d_{0} a_{p}^{2} \widetilde{\alpha}_{2} \overline{\widetilde{\alpha}}_{3}, d_{0} a_{p}^{2} \widetilde{\alpha}_{3} \overline{\widetilde{\alpha}}_{1}, d_{0} \alpha_{p}^{2} \widetilde{\alpha}_{1} \overline{\widetilde{\alpha}}_{2}\right) .
$$

Since $v\left(\vartheta_{p}^{\#}\right)=v\left(\vartheta_{p}\right)^{-1}$, another basis for $\psi_{p}\left(\vartheta_{L_{p}}\right)$ over $D_{p}$ is

$$
\left(1, \delta^{-1} \widetilde{\alpha}_{2} \overline{\widetilde{\alpha}}_{3}, \delta^{-1} \widetilde{\alpha}_{3} \overline{\widetilde{\alpha}}_{1}, \delta^{-1} \widetilde{\alpha}_{1} \overline{\widetilde{\alpha}}_{2}\right) \text {. }
$$

Let

$$
\alpha_{0}^{\prime}=1, \alpha_{1}^{\prime}=\delta^{-1} \widetilde{\alpha}_{2} \overline{\tilde{\alpha}}_{3}, \alpha_{2}^{\prime}=\delta^{-1} \widetilde{\alpha}_{3} \overline{\widetilde{\alpha}}_{1}, \alpha_{3}^{\prime}=\delta^{-1} \widetilde{\alpha}_{1} \overline{\widetilde{\alpha}}_{2} .
$$

If we show that $B\left(\alpha_{i}^{\prime}, \widetilde{\alpha}_{j}\right)=\delta_{i j}$ (Kronecker symbol), the result will follow by the uniqueness of the dual basis. The only difficulty lies in showing that $B\left(\alpha_{i}^{\prime}, \tilde{\alpha}_{i}\right)=1, i=1,2,3$, and since $B\left(\alpha_{i}^{\prime}, \tilde{\alpha}_{i}\right)=-$ $\delta^{-1} B\left(\widetilde{\alpha}_{1} \widetilde{\alpha}_{2} \widetilde{\alpha}_{3}, 1\right)$, we need only prove that $B\left(\widetilde{\alpha}_{1} \widetilde{\alpha}_{2} \widetilde{\alpha}_{3}, 1\right)=-\delta\left(\widetilde{\alpha}_{0}, \widetilde{\alpha}_{1}, \widetilde{\alpha}_{2}, \widetilde{\alpha}_{3}\right)$. Using the relationships in (2), a computation shows that

$$
B\left(\widetilde{\alpha}_{1} \widetilde{\alpha}_{2} \widetilde{\alpha}_{3}, 1\right)=\operatorname{det} T \cdot B\left(\gamma_{1} \gamma_{2} \gamma_{3}, 1\right) \text {. }
$$

From (3),

$$
B\left(\widetilde{\alpha}_{1} \widetilde{\alpha}_{2} \widetilde{\alpha}_{3}, 1\right)=\frac{\delta\left(\tilde{\alpha}_{0}, \widetilde{\alpha}_{1}, \widetilde{\alpha}_{2}, \widetilde{\alpha}_{3}\right)}{N\left(\gamma_{1}\right) N\left(\gamma_{2}\right)} \cdot B\left(\gamma_{1} \gamma_{2} \gamma_{3}, 1\right)
$$

Now,

$$
B\left(\gamma_{1} \gamma_{2} \gamma_{3}, 1\right)=B\left(\gamma_{1} \gamma_{2} \gamma_{1} \gamma_{2}, 1\right)=-B\left(\gamma_{1}^{2} \gamma_{2}^{2}, 1\right)=-N\left(\gamma_{1}\right) N\left(\gamma_{2}\right) .
$$

Hence $B\left(\widetilde{\alpha}_{1} \widetilde{\alpha}_{2} \widetilde{\alpha}_{3}, 1\right)=-\delta\left(\widetilde{\alpha}_{0}, \widetilde{\alpha}_{1}, \widetilde{\alpha}_{2}, \widetilde{\alpha}_{3}\right)$, and $\psi_{p}\left(\vartheta_{L_{p}}\right)=\vartheta_{p}$ for each $p$.

Since $\left(\psi\left(\vartheta_{L}\right)\right)_{p}=\psi_{p}\left(\vartheta_{L_{p}}\right)=\vartheta_{p}$ for every $p, \psi\left(\vartheta_{L}\right)=\vartheta$, and the theorem is proved.

In [6], $\S 4$, Peters has proved that a necessary and sufficient condition that a quaternion order $\vartheta$ be an image of a ternary lattice under Eichler's map is that $N\left(\vartheta^{\sharp}\right)^{-1}=\sqrt{v(\vartheta)}$. The following corollaries allow us to compare those orders which are images of ternary lattices under our correspondence and those which are images under Eichler's.

Corollary 5.2. Assume $\vartheta$ is the image of a lattice $L$ under Eichler's correspondence. Then $\vartheta$ is an image under our mapping if and only if $\widetilde{N}(L)$ is a square in the ideal class group of $D$.

Proof. From §3, (2)

$$
v(\vartheta)=d^{2} \tilde{N}(L)^{-6} A^{4}
$$

where $A$ is a fractional ideal and $d \in F$. If $\tilde{N}(L)$ is a square in the ideal class group of $D$, it is obvious that $\sqrt{v(\vartheta)}$ has the same property.

If $\vartheta$ is the image of an integral ternary lattice under the mapping defined in this paper, then for some ideal $B$ and for some $d_{0} \in F$, $v(\vartheta)=d_{0}^{2} B^{4}$. Hence 


$$
d^{2} \tilde{N}(L)^{-6} A^{4}=d_{0}^{2} B^{4},
$$

and

$$
\tilde{N}(L)=d d_{0}^{-1}\left[\tilde{N}(L)^{-1} A B^{-1}\right]^{2}=c_{0} C^{2},
$$

a square in the ideal class group of $D$.

Corollary 5.3. Assume $\vartheta$ is the image of a lattice $L$ under the correspondence defined in $\S 3$. Then $\vartheta$ is an image under Eichler's mapping if and only if $\tilde{N}(L)=D$.

Proof. It is a trivial consequence of (1), (2) of $\S 3$ that if $\tilde{N}(L)=$ $D$, then $\vartheta$ is the image of $L$ under Eichler's map.

Assuming $\vartheta$ is an image under both mappings, on the one hand $\sqrt{v(\vartheta)}=N\left(\vartheta^{\sharp}\right)^{-1}$, and on the other $\sqrt{v(\vartheta)}=d_{0} A^{2}$ for some fractional ideal $A$ and some $d_{0} \in F$. From the proof of Theorem 5.1, we may assume that $L=(A K)^{d_{0}}$ where $K=\left\{\omega \in \vartheta^{\sharp}: B(\omega, 1)=0\right\}$. From [6], $\S 3, N\left(\vartheta^{\sharp}\right)=N(K)$. Thus

$$
\tilde{N}(L)=d_{0} A^{2} N(K)=\sqrt{v(\vartheta)} \cdot N\left(\vartheta^{*}\right)=D .
$$

So it is easy to find orders which are realized as images of ternary lattices under our mapping but not under Eichler's. Furthermore, it is well-known that there exist Dedekind domains with (integral) ideals which are not squares in the ideal class group. The image under Eichler's correspondence of a lattice whose norm is such an ideal is not an image under our mapping.

Additionally, there are orders which are not images under either correspondence. To construct an example, let $Z$ denote the rational integers, let $D=Z[\sqrt{-10}]$, and let $A=5 Z+\sqrt{-10} Z$, an integral ideal which is not a square in the ideal class group of $D$ (see [1], p. 425). We note that $A^{2}=5 D$. Let $V$ be the ternary quadratic space with orthogonal basis $x_{1}, x_{2}, x_{3}$ satisfying

$$
\tilde{N}\left(x_{1}\right)=5, \tilde{N}\left(x_{2}\right)=\tilde{N}\left(x_{3}\right)=1 .
$$

In the even Clifford algebra of $V$, consider the order

$$
\vartheta=D \cdot 1+A x_{2} x_{3}+D x_{3} x_{1}+D x_{1} x_{2} .
$$

$\vartheta$ is not an image under either correspondence, since $\sqrt{v(\vartheta)}=5 \mathrm{~A}$, not a square in the ideal class group, and $N\left(\vartheta^{\sharp}\right)^{-1}=5 D \neq \sqrt{v(\vartheta)}$.

\section{REFERENCES}

1. Z. I. Borevich and I. R. Shafarevich, Number Theory, Academic Press, New York, 1966. 
2. M. Eichler, Quadratische Formen und orthogonale Gruppen, Springer-Verlag, BerlinGöttingen-Heidelberg, 1952.

3. I. Kaplansky, Submodules of quaternion algebras, Proc. London Math. Soc., (3) 19 (1969), 219-232.

4. O. T. O'Meara, Introduction to Quadratic Forms, Springer-Verlag, Berlin-GöttingenHeidelberg, 1963.

5. G. Pall, On generalized quaternions, Trans. Amer. Math. Soc., 59 (1946), 280-332.

6. M. Peters, Ternäre und quaternäre quadratische Formen und Quaternionenalgebren, Acta Arithmetica, 15 (1969), 329-365.

Received March 25, 1973 and in revised form February 12, 1974.

California State University, Los Angeles 



\section{PACIFIC JOURNAL OF MATHEMATICS}

\section{EDITORS}

RICHARD ARENS (Managing Editor)

University of California

Los Angeles, California 90024

\section{J. DUGUNDJI}

Department of Mathematics University of Southern California Los Angeles, California 90007

D. Gilbarg and J. Milgram

Stanford University

Stanford, California 94305

University of Washington
Seattle, Washington 98105

ASSOCIATE EDITORS
E. F, BECKENBACH
B. H. NEUMANN
F. WOLF
K. Yoshida

\section{SUPPORTING INSTITUTIONS}

\author{
UNIVERSITY OF BRITISH COLUMBIA \\ CALIFORNIA INSTITUTE OF TECHNOLOGY \\ UNIVERSITY OF CALIFORNIA \\ MONTANA STATE UNIVERSITY \\ UNIVERSITY OF NEVADA \\ NEW MEXICO STATE UNIVERSITY \\ OREGON STATE UNIVERSITY \\ UNIVERSITY OF OREGON \\ OSAKA UNIVERSITY
}

\author{
UNIVERSITY OF SOUTHERN CALIFORNIA \\ STANFORD UNIVERSITY \\ UNIVERSITY OF TOKYO \\ UNIVERSITY OF UTAH \\ WASHINGTON STATE UNIVERSITY \\ UNIVERSITY OF WASHINGTON \\ * * * * \\ AMERICAN MATHEMATICAL SOCIETY \\ NAVAL WEAPONS CENTER
}

The Supporting Institutions listed above contribute to the cost of publication of this Journal, but they are not owners or publishers and have no responsibility for its content or policies.

Mathematical papers intended for publication in the Pacific Journal of Mathematics should be in typed form or offset-reproduced, (not dittoed), double spaced with large margins. Underline Greek letters in red, German in green, and script in blue. The first paragraph or two must be capable of being used separately as a synopsis of the entire paper. Items of the bibliography should not be cited there unless absolutely necessary, in which case they must be identified by author and Journal, rather than by item number. Manuscripts, in duplicate if possible, may be sent to any one of the four editors. Please classify according to the scheme of Math. Rev. Index to Vol. 39. All other communications to the editors should be addressed to the managing editor, or Elaine Barth, University of California, Los Angeles, California, 90024.

100 reprints are provided free for each article, only if page charges have been substantially paid. Additional copies may be obtained at cost in multiples of 50 .

The Pacific of Journal Mathematics is issued monthly as of January 1966. Regular subscription rate: $\$ 72.00$ a year (6 Vols., 12 issues). Special rate: $\$ 36.00$ a year to individual members of supporting institutions.

Subscriptions, orders for back numbers, and changes of address should be sent to Pacific Journal of Mathematics, 103 Highland Boulevard, Berkeley, California, 94708.

PUBLISHED BY PACIFIC JOURNAL OF MATHEMATICS, A NON-PROFIT CORPORATION

Printed at Kokusai Bunken Insatsusha (International Academic Printing Co., Ltd.), 270, 3-chome Totsuka-cho, Shinjuku-ku, Tokyo 160, Japan.

Copyright (C) 1973 by Pacific Journal of Mathematics Manufactured and first issued in Japan 


\section{Pacific Journal of Mathematics}

\section{Vol. 53, No. $2 \quad$ April, 1974}

Kenneth Abernethy, On characterizing certain classses of first countable spaces by

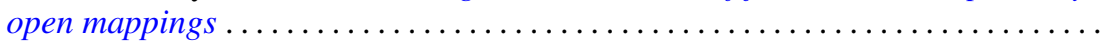

Ross A. Beaumont and Donald Lawver, Strongly semisimple abelian groups .......

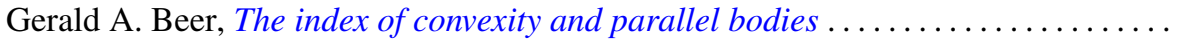

Victor P. Camillo and Kent Ralph Fuller, On Loewy length of rings ..............

Stephen LaVern Campbell, Linear operators for which $T^{*} T$ and $T T^{*}$ commute.

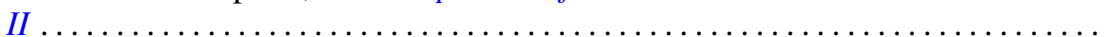

Charles Kam-Tai Chui and Philip Wesley Smith, Characterization of a function by

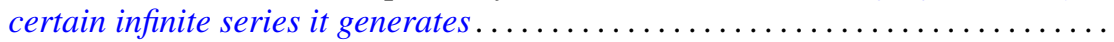

Allan L. Edelson, Conjugations on stably almost complex manifolds . ...........

Patrick John Fleury, Hollow modules and local endomorphism rings . . ..........

Jack Tilden Goodykoontz, Jr., Connectedness im kleinen and local connectedness in

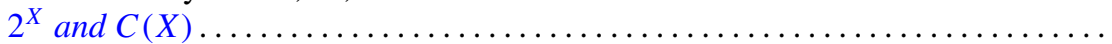

Robert Edward Jamison, II, Functional representation of algebraic intervals .......

Athanassios G. Kartsatos, Nonzero solutions to boundary value problems for

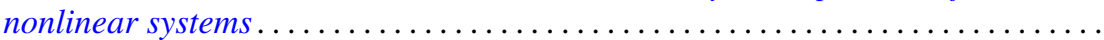

Soon-Kyu Kim, Dennis McGavran and Jingyal Pak, Torus group actions on simply

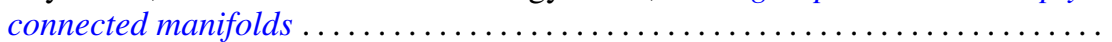

David Anthony Klarner and R. Rado, Arithmetic properties of certain recursively

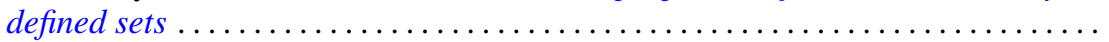

Ray Alden Kunze, On the Frobenius reciprocity theorem for square-integrable

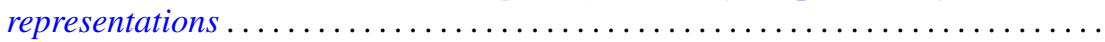

John Lagnese, Existence, uniqueness and limiting behavior of solutions of a class of differential equations in Banach space...

Teck Cheong Lim, A fixed point theorem for families on nonexpansive mappings Lewis Lum, A quasi order characterization of smooth continua

Andy R. Magid, Principal homogeneous spaces and Galois extensions . .

Charles Alan McCarthy, The norm of a certain derivation ..... . .

Louise Elizabeth Moser, On the impossibility of obtaining $S^{2} \times S^{1}$ by elementary surgery along a knot. .

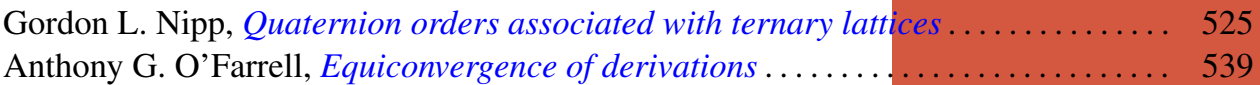

Dorte Olesen, Derivations of $A W^{*}$-algebras are inner . . . . . . . . . . . . . . . 555

Dorte Olesen and Gert Kjærgaard Pedersen, Derivations of $C^{*}$-algebras have

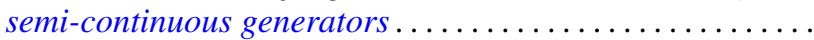

Duane O’Neill, On conjugation cobordism.

Chull Park and S. R. Paranjape, Probabilities of Wiener paths crossing differentiable

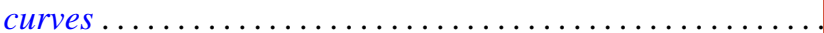

Edward Ralph Rozema, Almost Chebyshev subspaces of $L^{1}(\mu$;

Lesley Millman Sibner and Robert Jules Sibner, A note on the Atiyah-Bott fixed

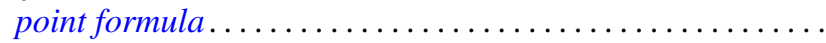

Betty Salzberg Stark, Irreducible subgroups of orthogonal groups generated by

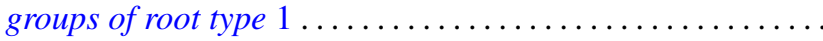

N. Stavrakas, A note on starshaped sets, $(k)$-extreme points and the half ray

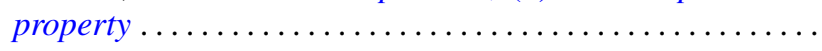

Carl E. Swenson, Direct sum subset decompositions of $Z \ldots \ldots$ 